\title{
Reliability-based robust design optimization of laminated composite structures
}

\author{
Zhao Weitao ${ }^{\text {* }}$, Zhang Xizhi \\ Key Laboratory of Liaoning Province for Composite Structural Analysis of Aerocraft and Simulation, \\ Shenyang Aerospace University, Shenyang 110136, China \\ a zhwt201@163.com
}

Keywords: composite materials, robust design, reliability, optimization

Abstract. The probability approach is the most popular way to quantify uncertain parameters and perform reliability analysis. The problems of structural optimization and robust design for laminated composite structures with reliability constraints are addressed in this paper. A new methodology to determine the optimal robust configuration of laminated composite structures with reliability constraints is proposed. In the proposed method, the criterion of Tsai-Wu is adopt to determine the failure of composite materials, the reliability analysis is performed by using Monte Carlo simulation, and the multi-objective optimization involved in the classical reliability-based robust design can be transformed into the single-objective optimization by using two-leveloptimization algorithm. A numerical example is given, it is shown that the proposed method is effective and feasible.

\section{Introduction}

Aeronautic applications of composite materials have increased due to their excellent specific stiffness, low weight, and designability. The number of layers, fiber orientation angles, thickness of each layer, and the sequence of layers can be changed to optimize laminated composite structures. Lots of unavoidable uncertainties exist when laminated composite structures are generally used under special and severe circumstances. Thus it is necessary to take the uncertainties into consideration when designing these structures. Consequently, reliability of optimized designs becomes especially important in the field of laminated composite structures [1]. Reliability-based design optimization (RBDO) of composite structures is currently a very important area of research[2-5].

In the actual production, the design parameters may appear error to influence the objective function. In traditional optimization, this influence is not considered, and robust design methods can solve this problem. Robust design optimization of composite structures is another emerging area of research that is thought of as an alternative design to RBDO. Robust design is to improve product quality by minimizing the uncertainty effects in structural response. Robust design optimization of composite structures under probabilistic constraints is a very important due to uncertainties associated with physical properties of fiber-reinforced composites.

The weighted methods are often used in most robust design methods [1-6]. Due to the weighted coefficient is difficult to determine, also there is no clear theory basis. Thus in this paper, the two-level optimization algorithm is presented to solve the problem. In the proposed method, the multi-objective optimization involved in the classical reliability-based robust design can be transformed into the single-objective optimization.

\section{Robust design optimization}

Weighted method. The basic mathematical expression of weighted methods is

$$
\begin{array}{lc}
\min & f(\mathbf{X})=\eta_{1} W_{1}(\mathbf{X})+\eta_{2} W_{2}(\mathbf{X}) \\
\text { s.t. } & \beta \geq \beta_{0} \\
& \mathbf{X} \in\left[\mathbf{X}^{L}, \mathbf{X}^{U}\right]
\end{array}
$$




$$
W_{1}(\mathbf{X})=\sum_{i=1}^{m} h_{i}, \quad W_{2}(\mathbf{X})=\sum_{i=1}^{n} \frac{\partial P_{f}}{\partial \mu_{x_{i}}}
$$

where $\beta_{0}$ is the prescribed reliability index, $W_{1}(\mathbf{X})$ is a function describing the structural weight, $m$ is the layer number of laminated plates. $W_{2}(\mathbf{X})$ is a function describing the structural sensitivity, $n$ is the number of random variables. $\eta_{1}$ and $\eta_{2}$ are weighting coefficients, $h_{i}$ is the thickness of $i$ th layer, $P_{f}$ is failure probability, and $\mu_{x_{i}}$ is mean value of random variable $x_{i}$, respectively.

Two-level optimization algorithm. The classical robust design optimization is a bi-objective optimization. It is difficult to determine the weighted coefficients. In this study, the two-level optimization algorithm is presented, as follows:

$$
\begin{array}{ccc} 
& \min & f(\mathbf{X})=W_{1}(\mathbf{X}) \\
\text { Step 1: } & \text { s.t. } & \beta \geq \beta_{0} \\
& & \mathbf{X} \in\left[\mathbf{X}^{L}, \mathbf{X}^{U}\right] \\
& \min & f(\mathbf{X})=W_{2}(\mathbf{X}) \\
& \text { s.t. } & \beta \geq \beta_{0} \\
\text { Step 2: } & & W_{1}(\mathbf{X}) \leq(1+k) W_{0} \\
& & \mathbf{X} \in\left[\mathbf{X}^{L}, \mathbf{X}^{U}\right]
\end{array}
$$

where $W_{0}$ is the optimal solution obtained by using reliability-based design optimization. In practical application, in order to get the best robustness solution, $k$ is coefficient to control structural weight.

\section{Failure criterion}

Tsai-Wu criterion is the most widely used because it best represents quite well the actual behavior of laminated composite structures. It can be expressed as

$$
F_{11} \sigma_{1}^{2}+F_{22} \sigma_{2}^{2}+2 F_{12} \sigma_{1} \sigma_{2}+F_{66} \tau_{12}^{2}+F_{1} \sigma_{1}+F_{2} \sigma_{2}=1
$$

where

$$
F_{11}=\frac{1}{X_{t} X_{c}}, F_{22}=\frac{1}{Y_{t} Y_{c}}, F_{1}=\frac{1}{X_{t}}-\frac{1}{X_{c}}, F_{2}=\frac{1}{Y_{t}}-\frac{1}{Y_{c}}, F_{66}=\frac{1}{S^{2}}, F_{12}=-\frac{1}{2} \sqrt{F_{11} F_{22}}
$$

where $\sigma_{1}, \sigma_{2}, \tau_{12}$ is normal stress and shear stress, $X_{t}$ and $X_{c}$ are tensile and compressive strength in the transverse direction, $Y_{t}$ and $Y_{c}$ are tensile and compressive strength in the longitudinal direction, respectively.

The limit state function in terms of Tsai-Wu failure criterion can be expressed as

$$
G(\mathbf{X})=1-\left(F_{11} \sigma_{1}^{2}+F_{22} \sigma_{2}^{2}+2 F_{12} \sigma_{1} \sigma_{2}+F_{66} \tau_{12}^{2}+F_{1} \sigma_{1}+F_{2} \sigma_{2}\right)
$$

It defines the failure domain by $G(\mathbf{X}) \leq 0$.

\section{Reliability analysis and sensitivity}

The Monte Carlo simulation is used to calculate the structural reliability. It can be expressed as

$$
P_{f}=\frac{N_{f}}{N}
$$

where $N$ is the number of sample points. $N_{f}$ is the number of sample points in the failure region.

Structural sensitivity can be expressed as [7]

$$
W\left(x_{i}\right)=\frac{\partial P_{f}}{\partial \mu_{x_{i}}}=P_{f} E\left[\frac{z_{i}}{\sigma_{x_{i}}}\right]_{\Omega}=\frac{P_{f}}{\sigma_{x_{i}}} E\left[z_{i}\right]_{\Omega}
$$

where $E\left[z_{i}\right]_{\Omega}$ is mean value of $z_{i}$ in failure region, $z_{i}$ are independent standard normal variables. 


\section{Genetic algorithm}

Genetic algorithms have been used in a wide variety of fields to evolve solutions to problems as difficult as or more difficult than those faced by human designers. Genetic algorithms have proven to be an enormously powerful and successful problem-solving strategy[8]. Thus, in this paper, the standard genetic algorithm is used to optimize structures.

\section{Numerical examples}

This example deals with the minimization of the total thickness of a laminated composite plate with linear behavior[8]. The total number of layers is $N$ and the thickness of layer $i$ is $h_{i}(i=1,2,3,4)$. The constraint is the minimum reliability index which is 3.0. The reliability index is calculated by using Monte Carlo simulation and the limit state function considered here is the Tsai-Wu failure criterion. The robust design optimization by using the proposed method be expressed as

$$
\min f(\mathbf{X})=W_{1}(\mathbf{X})=\sum_{i=1}^{N} h_{i}(i=1,2,3,4)
$$

Step 1: s.t. $\quad \beta \geq 3.0$

$$
\begin{array}{rc}
\min \quad f(\mathbf{X})=W_{2}(\mathbf{X})=\sum_{i=1}^{n} \frac{\partial P_{f}}{\partial \mu_{x_{i}}} \\
\text { Step 2: s.t. } \quad \beta \geq 3.0 \\
W_{1}(\mathbf{X}) \leq(1+k) W_{0} \\
\mathbf{X} \in[0,5 \mathrm{~mm}]
\end{array}
$$

The fiber orientation angle of each ply of the laminated composite plate with four layers remains constant and according to the distribution $\left[0^{\circ} / 45^{\circ} / 45^{\circ} / 0^{\circ}\right]$, as shown in Fig. 1 . The material used here was Graphite/Epoxy (T300/5208). Table 1 presents the deterministic mechanical properties. In this example nine random variables are considered, where four variables are the applied loads $N_{1}, N_{2}, N_{12}$ and $M_{1}$, arranged as shown in Fig. 2, and five variables are strength parameter. In Fig. 2, $(x, y)$ is the fiber orientation system and (1, 2 ) is the global system. The statistical properties of random variables are listed in Table 2, where COV means the coefficient of variation.

Table 1 Deterministic mechanical properties

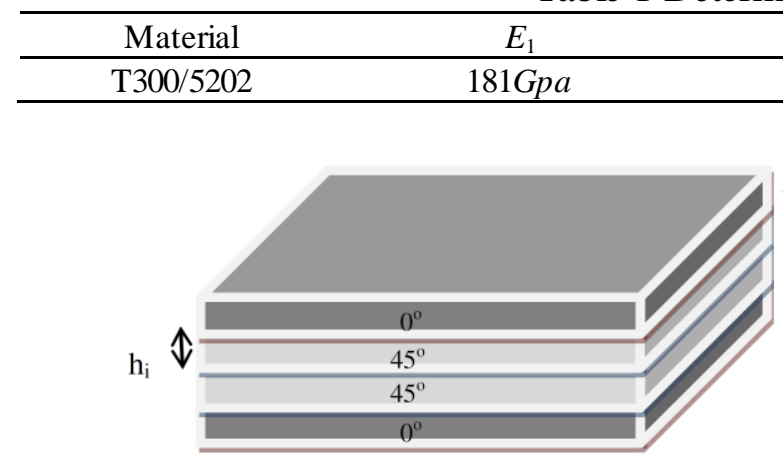

Fig. 1. Laminated composite plate

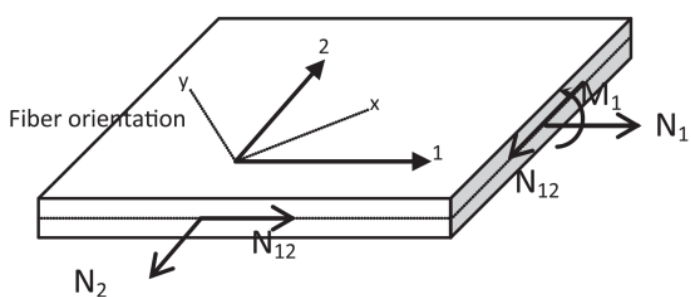

Fig. 2. Loads 
Table 2 Statistical properties of random variables

\begin{tabular}{cccccc}
\hline No. & Symbol & Unit & Mean Value & Coeff.of variation & Distribution type \\
\hline 1 & $N_{1}$ & $\mathrm{KN} / \mathrm{m}$ & 100 & 0.20 & Lognormal \\
2 & $N_{2}$ & $\mathrm{KN} / \mathrm{m}$ & 200 & 0.20 & Lognormal \\
3 & $N_{12}$ & $\mathrm{KN} / \mathrm{m}$ & 40 & 0.20 & Lognormal \\
4 & $M_{1}$ & $\mathrm{~N} \cdot \mathrm{m} / \mathrm{m}$ & 0.1 & 0.20 & Lognormal \\
5 & $X_{t}$ & $\mathrm{Mpa}$ & 1500.0 & 0.20 & Lognormal \\
6 & $X_{c}$ & $\mathrm{Mpa}$ & 1500.0 & 0.20 & Lognormal \\
7 & $Y_{t}$ & $\mathrm{Mpa}$ & 40.0 & 0.20 & Lognormal \\
8 & $Y_{c}$ & $\mathrm{Mpa}$ & 246.0 & 0.20 & Lognormal \\
9 & $S$ & $\mathrm{Mpa}$ & 68.0 & 0.20 & Lognormal \\
\hline
\end{tabular}

The results obtained by using the proposed method are listed in Table 3. As seen from Table 3, comparing with the results of $k=0$ and $k=0.1$, the total thickness of a laminated composite plate increases only $3 \%$ when $k=0.1$, however, the reliability sensitivity decrease rapidly when $k=0.1$. Comparing with the results of $k=0$ and $k=0.2, k=0.3$ and $k=0.4$, the total thickness of a laminated composite plate increases rapidly, however, the reliability sensitivity decrease slowly. Thus, we can choose the best results according to actual engineering, for this example, the results of $k=0.1$ is selected as the optimum solution.

Table 3 Results obtained by using the proposed method

\begin{tabular}{cccccccc}
\hline value & $h_{1}(\mathrm{~mm})$ & $h_{2}(\mathrm{~mm})$ & $h_{3}(\mathrm{~mm})$ & $h_{4}(\mathrm{~mm})$ & $W_{1}(\mathbf{X})$ & $\beta$ & $W_{2}(\mathbf{X})$ \\
\hline$k=0$ & 2.5445 & 1.6795 & 1.7046 & 1.8394 & 7.7680 & 3.0904 & $9.48 \times 10^{-6}$ \\
$k=0.1$ & 1.3881 & 2.4615 & 2.9543 & 1.1931 & 7.9970 & 3.7694 & $1.85 \times 10^{-9}$ \\
$k=0.2$ & 1.7851 & 2.3412 & 2.5236 & 2.3540 & 9.0039 & 3.8694 & $5.49 \times 10^{-10}$ \\
$k=0.3$ & 3.0245 & 2.3355 & 2.4231 & 2.0053 & 9.7884 & 4.1294 & $5.70 \times 10^{-11}$ \\
$k=0.4$ & 1.6838 & 3.3583 & 2.1161 & 3.0489 & 10.2071 & 4.2861 & $3.42 \times 10^{-11}$ \\
\hline
\end{tabular}

\section{Summary}

In this study, a new methodology to determine the optimal robust configuration of laminated composite structures with reliability constraints is proposed. In the proposed method, the two-level optimization algorithm is proposed, the multi-objective optimization involved in the classical reliability-based robust design can be transformed into the single-objective optimization. All results obtained by using the proposed method are feasible solutions, we can choose the best results according to actual engineering.

\section{Acknowledgements}

The research described in this paper was financially supported by the Aerospace Science Foundation of China (2013ZA54004) and the Education Department of Liaoning Province Research Projects of China (L2014072).

\section{References}

[1] N. Kogiso, Y. Murotsu, Reliability analysis of laminated composite plate with initial imperfection, Trans. JSME, Ser. A. 66(2000)1483-1490.

[2] M. Miki, Optimum fiber orientation angle of multi-axially laminated composites based on reliability, Reliab. Eng. Syst. Safe. 66(2007)279-285.

[3] C.C. Antinio, L.N. Hoffbauer, An approach for reliability-based robust design optimization of angle-ply composites, Compos. Struct. 90(2009):53-59.

[4] E. Reneud, Robustness design through minimum sensitivity, Adv. Des. Automat. 121(2007)278- 287.

[5] C. Boyer, A. Béakou, M.Lemaire, Design of a composite structure to achieve a specified reliability level, Reliab. Eng. Syst. Safe. 56(1997):273-283. 
[6] S. Adali, F. Lene, G. Duvaut, V. Chiaruttini, Optimization of laminated composites subject to uncertain buckling loads, Compos. Struct. 62(2003)261-269.

[7] Z. Weitao, Z.xu, Based on Monte Carlo method of structure system reliability calculation and sensitivity analysis, J. Comput. Mech. 28(2011) 200-204.

[8] H.M.Gomes, A.M.Awruch, P.A.M. Lopes, Reliability based optimization of laminated composite structures using genetic algorithms and Artificial Neural Networks, Struct. Safe. 33(2011):186-195. 